\title{
Link Selection for Point-to-Point 60GHz Networks
}

\author{
Candy Yiu \\ Department of Computer Science \\ Portland State University \\ Portland, OR 97207 \\ Email: candy@cs.pdx.edu
}

\author{
Suresh Singh \\ Department of Computer Science \\ Portland State University \\ Portland, OR 97207 \\ Email: singh@cs.pdx.edu
}

\begin{abstract}
GHz is well-suited for device-to-device communication and for general in-home applications due to its huge available bandwidth. However, links at this frequency are easily degraded by environmental conditions. Indeed, the poor multipath makes it necessary to maintain good LoS (Line of Sight) paths between communicating pairs of nodes. In this paper we analyze the problem of link breakage and degradation in point-to-point $60 \mathrm{GHz}$ networks. We propose using repeaters to provide alternate paths between communicating nodes when the direct path degrades. The decision on which path to use is driven by the goal of maximizing data rate per connection as well as overall throughput in the network. Using extensive simulations we show that by carefully using repeaters, we can maintain Gbps rates for each pair of communicating nodes in indoor spaces. We develop an efficient distributed algorithm for allocating repeaters to links as needed and explicitly deal with the problem of interference caused by the repeaters themselves. Finally, we study the scalability of the solution with increasing numbers of communicating pairs and show that Gbps rates can be maintained even when there are as many as eight communicating pairs in a small $10 \mathrm{mx10m}$ room.
\end{abstract}

\section{INTRODUCTION}

With its large available bandwidth and restricted propagation properties, $60 \mathrm{GHz}$ promises to be the communication technology of choice for the home and office. However, while its restricted propagation allows efficient spatial reuse of the spectrum, it also makes links highly susceptible to obstructions and LoS (Line of Sight) interference. This paper considers a point-to-point model of $60 \mathrm{GHz}$ networking where pairwise communication between nodes is the predominant form of communication - unlike the standard WLAN model where every node talks to an access point alone. In Figure 1(a) we show a typical room with several communicating pairs of devices. The wall-mounted display is showing a home movie that is streamed from a camera while a pair of users has set up a separate link to communicate and a third link connects a media server to a user's music headset. In the context of this network model, we note that there exists a problem with link breakage or degradation because of interference. For instance, consider Figure 1(b) where a person has walked into the room. In this position, the presence of the user can break or severely degrade link 2 between the video camera and the display. Figure 1(c) shows a case where a new user enters the room and sets up a link with another device. This causes link 3 to degrade due to interference. In both of these cases, the existing links need to be repaired so as to maintain the needed data rate. This paper studies the problem of selecting links that meet the needed data rate for pairwise communication in such indoor environments.

The approach we investigate relies on the existence of one or more repeaters strategically placed about the room. These devices provide alternative routes between communicating pairs. For the case illustrated in Figure 1(b), a new 2-hop link can be established between the video camera and display that is obstruction free as shown in Figure 2. Likewise, when the quality of link 3 in Figure 1(c) degrades due to LoS interference, the link can be recreated via another repeater, also as shown in Figure 2. These examples illustrate the benefits of repeaters but it should be clear that in more complex environments, the placement and selection of repeaters is a non-trivial problem. Indeed, it is quite possible that using a repeater to fix one link may well degrade another existing link. Also, if several repeaters are needed simultaneously, the assignment of repeaters to links is key to reaching the required data rate per pair-wise connection as well as system throughput. In this paper we study the problem of repairing links via repeaters and propose an algorithm for the assignment problem. We conduct a detailed analysis of the solution and study how it scales with increasing numbers of links.

The remainder of the paper is organized as follows. Section II presents related work. The subsequent section describes the system model used and outlines the challenges in using repeaters. Section IV describes our algorithm for assigning repeaters and the performance of the solution is studied in detail in section V. We conclude in section VI.

\section{RELATED WORK}

A unique characteristic of $60 \mathrm{GHz}$ signals is that they are well-absorbed by oxygen and by several commonly used materials [9], [5], [6], [4], [8] leading to the absence of a rich multipath environment. For instance, [2] reports on propagation measurements at $60 \mathrm{GHz}$ and $2.5 \mathrm{GHz}$. They note that large-scale propagation for $60 \mathrm{GHz}$ can be modeled as free space (a measured path loss exponent of 2.1 in buildings) as was also noted by [1]. Other results of these and other measurement studies [12] note the small RMS delay spread for $60 \mathrm{GHz}$ indicating little multipath. [14] presents a detailed measurement study of $60 \mathrm{GHz}$ propagation in indoor environments with particular attention to multipath. They note that secondorder and higher-order reflections are highly attenuated and 


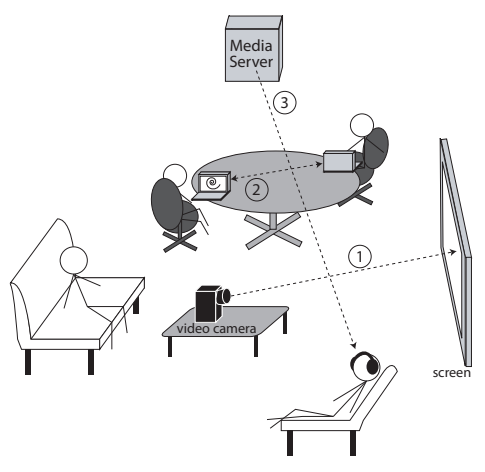

(a) A typical use environment showing 3 pairs of communicating pairs.



(b) Link 1 between video camera and screen degrades due to obstruction (person).

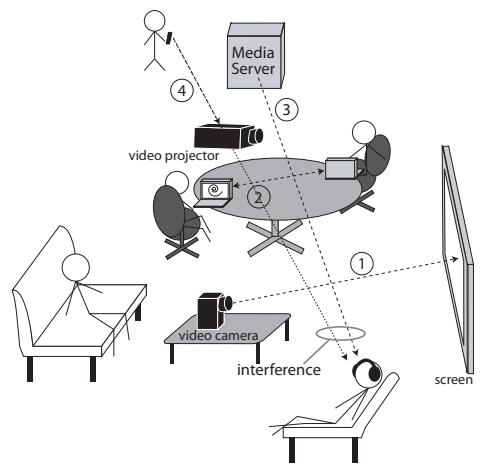

(c) Interference caused at the user of link 3 by the appearance of new link 4 .

Fig. 1. Problem with obstructions and interference.

negligible. Penetration loss through walls in the building are very high with many examples of over $35 \mathrm{~dB}$. This makes the Line of Sight (LoS) path the predominant signal path between communicating pairs of nodes. The benefit of this behavior is the potential for highly efficient spatial reuse. However, the drawbacks are many and form the topic of the present paper.

The problem of channel allocation between pairs of communicating nodes in a $60 \mathrm{GHz}$ WLAN has not been studied much. One exception is [10] where the authors present a MAC design for a multihop $60 \mathrm{GHz}$ WPAN. In their model, every node uses highly directional antennas and high transmit power to maintain a network-wide rate of $2 \mathrm{Gbps}$. However, the paper does not consider the attenuation due to different materials

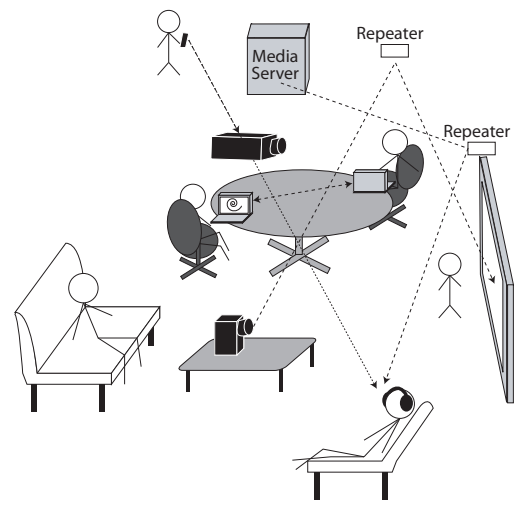

Fig. 2. Repairing links using repeaters.

that may obstruct the signal path (including the human body). Indeed, they do not consider the problem of jointly selecting links and data rates in the WLAN as a whole.

Rate adaptation refers to the problem of selecting an appropriate modulation for current channel conditions at a receiver. In the context of 802.11, several algorithms have been developed for dynamically changing rates based on inferred channel information, see [3] and [13]. In the context of 60 $\mathrm{GHz}$, the problem takes on a different flavor. Since we have multiple links between a pair of nodes (direct or via reflectors), rate adaptation (i.e., modulation) and link selection can be viewed as joint problems. In our work here, we select links such that the maximum rate of $1 \mathrm{Gbps}$ is sustainable.

\section{System ModeL}

Figure 1 illustrates the usage model we consider. In general, we assume there are $n$ pairs of communicating nodes and some number $k$ of repeaters deployed about the room (the repeaters may well be other idle nodes that are tasked to aid active connections). All the nodes and repeaters are assumed to be equipped with smart antennas, each with $M$ antenna elements. The nodes and repeaters can beamform in any direction. Further, since a repeater serves to connect a communicating pair of nodes, we assume that it can simultaneously communicate with both the nodes that form the end-points of the link. Thus, the repeaters may be implemented either as store and forward nodes that receive packets on one link and then forward them on the other or as cut-through devices where the incoming signal is not decoded but simply forwarded on the outgoing link. We note that the analysis in this paper is valid for either model.

The problem we consider can be summarized as follows: given $n$ communicating node pairs and $k$ repeaters, how can we establish $n$ connections such that data rates are maximized for each pair? The problem is non-trivial because of interference and the existence of obstructions in the LoS path between pairs of communicating nodes. Figure 3 illustrates a simple case where one link interferes with another, thus reducing the 


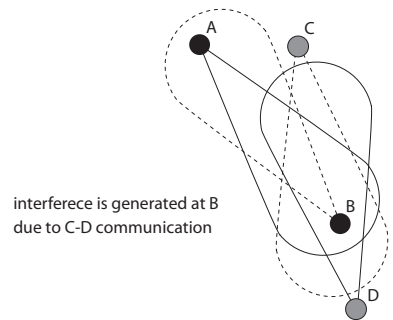

Fig. 3. Degradation of a link due to interference from another.

data rate for that link ${ }^{1}$. As we can see, the transmissions from node A to B will generate an interfering signal at node D thus degrading the SINR (Signal to Interference and Noise Ratio) and the data rate at $\mathrm{D}$.

One can argue that with narrow enough beams, the amount of such interference can be eliminated or made negligible. In order to study this assertion further, we ran Matlab simulations for random node placements and measured the interference. For a given $n$, we randomly uniformly place each node of that link somewhere within a room of size $10 \mathrm{mx} 10 \mathrm{~m}$. Each node is assumed to have a linear array with $M=20$ antenna elements. We use standard expressions for computing the array factor (AF) [7],

$$
A F=\sum_{i=1}^{M} e^{j(i-1) k d\left(\sin \theta-\sin \theta_{0}\right)}
$$

where $k=2 \pi / \lambda$ and $d$ is the antenna element spacing. $\theta_{0}$ is the angle at which we are forming the main beam and $\theta$ is the angle at which we are computing the array factor. $\lambda$ is the wavelength at $60 \mathrm{GHz}$.

Each transmitter beamforms towards its receiver and each receiver beamforms towards its transmitter. At each receiver we calculate the total interference generated from each of the other $n-1$ transmitters. To do this accurately, we use a detailed $60 \mathrm{GHz}$ propagation model (described in [15]) that we have built in Matlab. The model uses measured attenuation data for transmissions through and reflections off surfaces to predict the signal strength at each point in the room. Thus, at each receiver, we measure the sum total of direct as well as reflected signal components that act as interference. The path loss exponent is assumed to be 2.1 following [12]. The simulation model also accounts for inter-symbol interference due to multipath between a communicating pair. We use a symbol time of $1.5625 \mathrm{~ns}$ for the $640 \mathrm{MHz}$ channel considered. Other system parameters used are given in Tables I and II.

Figure 4(a) plots the number of links that break due to interference as a function of $n$. A link breaks if the SINR at the receiver is low enough that none of the $60 \mathrm{GHz}$ rates can be supported. We see that when there are 10 links, on average 2 break and as many as 5 can break depending on the actual positions of the communicating nodes. This is despite the fact that

\footnotetext{
${ }^{1} \mathrm{We}$ emphasize that our focus in this paper is on the data rates achievable using appropriate resource allocation algorithms at the Physical layer. We assume that collision events (i.e., where two or more transmissions collide at a receiver) are handled by a MAC layer.
}

\begin{tabular}{|l|l|}
\hline Room dimensions & $10 \mathrm{~m} \times 10 \mathrm{~m} \times 3 \mathrm{~m}$ \\
Transmit power & $10 \mathrm{dBm}$ \\
Bandwidth & $640 \mathrm{MHz}$ \\
Modulation & Table II \\
No. antenna elements & $M=20$ \\
\hline
\end{tabular}

TABLE I

EXPERIMENTAL PARAMETERS.

\begin{tabular}{|c|c|c|c|}
\hline Modulation & Code Rate & Min $E_{b} / N_{0}$ for $P_{b} \leq 10^{-6}$ & Rate $($ Mbps $)$ \\
\hline 64-QAM & $3 / 4,1 / 2$ & $22.65 \mathrm{~dB}$ & 1080,960 \\
16-QAM & $3 / 4,1 / 2$ & $19.1 \mathrm{~dB}$ & 720,480 \\
QPSK & $3 / 4,1 / 2$ & $16.7 \mathrm{~dB}$ & 360,240 \\
BPSK & $3 / 4,1 / 2$ & $11.45 \mathrm{~dB}$ & 180,120 \\
\hline
\end{tabular}

TABLE II

BIT PER SECOND FOR DIFFERENT MODULATION SCHEMES [11].

we use highly directional beams for communication. The effect of interference is better illustrated when we look at Figure 4(b) where we plot the per-link throughput (max and average). Here, as the number of links increases, the average throughput starts falling linearly reaching a low of 600Mbps with 20 links. The inescapable conclusion is that, despite narrow beams and the constrained propagation behavior of $60 \mathrm{GHz}$ signals, there is sufficient interference between simultaneous links to cause communication failure. Hence the case for repeaters is welljustified.

\section{A. Problem Formulation}

Let $p_{1}, p_{2}, \cdots, p_{n}$ denote the $n$ communicating pairs of nodes and let $k$ be the number of available repeaters. An allocation $\vec{a}$ is a $n$-tuple $\left(a_{1}, a_{2}, \cdots, a_{n}\right)$ where, $0 \leq a_{i} \leq k$ such that, $\forall a_{i}, a_{j}>0, i \neq j, a_{i} \neq a_{j}$. The interpretation is that if $a_{i}=0$ then the pair $p_{i}$ communicates directly. If $a_{i}=l$ then the pair sets up a connection via repeater $l$. For a given allocation $\vec{a}$ let $\left\{r_{1}, r_{2}, \cdots, r_{n}\right\}$ be the data rates achieved by each pair of communicating nodes (after considering interference, attenuation, etc.) and let $r(\vec{a})=\min \left\{r_{1}, r_{2}, \cdots, r_{n}\right\}$. Define $\vec{a} *$ to be the optimal allocation if,

$$
r(\vec{a} *) \geq r(\vec{a}), \forall \vec{a} \in \mathcal{A}
$$

where $\mathcal{A}$ is the set of all possible allocations. The number of allocations can be written as,

$$
|\mathcal{A}|=\sum_{i=0}^{k}\left(\begin{array}{c}
k \\
i
\end{array}\right)\left(\begin{array}{c}
n \\
i
\end{array}\right)
$$

Observe that the optimal solution may only use $k^{\prime} \leq k$ repeaters. We note that the problem of finding $\vec{a} *$ can be shown to be NP-hard (we omit the proof here for space reasons).

The more realistic version of the problem is one where each pair of communicating nodes tries to optimize its performance independently of the other pairs. In this distributed version of the problem, the definition of optimum remains unchanged but the problem of finding the optimal solution is harder. In the next section we focus on this distributed problem and develop a simple solution to it. 




(a) Number of broken links

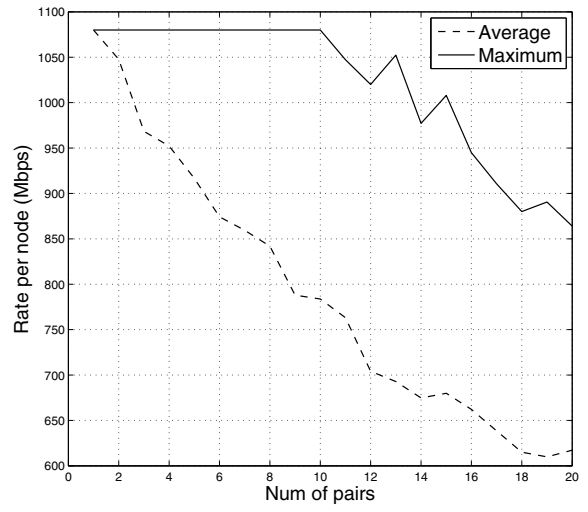

(b) Throughput

Fig. 4. Impact of interference.

\section{GREedy Algorithm}

We develop a distributed greedy algorithm for finding an allocation that, in most cases, achieves the optimal link allocation. The algorithm is iterative and works as follows:

1) Initially each link is set up directly between the two end-points.

2) Each pair computes the best achievable rate for each direction of communication.

3) If a link does not achieve $800 \mathrm{Mbps}$ rate (in either direction), it will randomly uniformly choose a free repeater.

4) The link is now set up via this repeater, and this is done by all the links that fall below 800 Mbps.

5) After this step, each link recomputes the achievable data rate. It is possible that a previously good link now shows degraded performance due to interference from a newly rerouted link. As previously, every link that falls below the $800 \mathrm{Mbps}$ threshold selects a new free repeater.

6) The algorithm iterates until no further improvement is seen in two consecutive steps. It is possible that the algorithm terminates with some pairs seeing data rates that are below the $800 \mathrm{Mbps}$ threshold.
Figure 5 illustrates the workings of this algorithm for a case when we have $n=6$ and $k$ is unrestricted (this is a screenshot of a visualization tool built on top of our Matlab simulator). The room is $10 \mathrm{mx} 10 \mathrm{~m}$ and all nodes as well as repeaters are at a height of $1 \mathrm{~m}$. In the figure, each of the six pairs is labeled 1- 6 and the repeaters that get used are numbered R1, R2, etc. Initially, each of the pairs sets up a direct connection between the two end-points using their smart antennas. The bottom two bar charts in the figure correspond to the four iterations of the algorithm where the SINR and Rate is shown at the end of each iteration for each of the six pairs of nodes. Each bar (in a set of four bars) is one iteration of the algorithm for a given node.

In the figure, we plot the minimum observed SINR for each pair as the first bar (of the four bars) and is labeled by ' $\mathrm{D}$ ' (this is the direct path). The achieved data rate for each of the pairs is shown in the bottom most plot. Pairs 3 and 4 have a low data rate of $600 \mathrm{Mbps}$ and they each re-route the connection via repeaters in the next step - pair 3 goes via R8 and pair 4 goes via R1. The new SINRs and data rates for the five pairs are shown as the second bar in each group of bars in the bottom two plots. As a result of this re-routing (pair 3 via R8 and pair 4 via R1), the SINR for pair 5 drops, as does its data rate. Pairs 1 and 2 also see a small degradation in SINR but the data rate remains high. In the next iteration, pair 4 switches from R1 to R4 and pair 5 now chooses to go via a repeater R7. This improves pair 5's data rate but pair 4 is still below threshold. Finally, pair 4 changes the repeater yet again and selects R3. At this point, all the pairs have a data rate greater than the threshold of $800 \mathrm{Mbps}$ and the algorithm terminates.

\section{EXPERIMENTAL EVALUATION}

The goal of the simulations is to understand the effectiveness of repeaters in mitigating link failure. The metrics we used to study this question are:

- Data rate achieved per user,

- Number of repeaters used to fix all link breakages,

- Percentage improvement in throughput when using repeaters.

In order to get a comprehensive understanding of how repeaters may help, we used a large number of node placements in our study. Specifically, we use a room of size $10 \mathrm{mx} 10 \mathrm{~m}$ within which we placed $2 n$ nodes randomly uniformly giving us $n$ links. We considered $n=4,5,6,7,8$. For each value of $n$ we randomly generated 1000 different configurations and studied the performance of our algorithm in each case. Repeaters are placed at grid locations within the room and we use 16 repeaters in all. Note that no more than $n$ repeaters will be used for a given $n$ since we only consider cases when a link is routed through at most one repeater. The case when the number of repeaters $k<n$ is a subset of the case when $k$ is unrestricted. For instance, if the number of repeaters used for a $n$ is $l$ then we know that using $k<l$ will result in $(k-l)$ broken links. 




Fig. 5. Illustration of the greedy algorithm.

Finally, we place the nodes and repeaters all at a height of $1 \mathrm{~m}$ above the floor. There are two reasons for this choice. First, in real deployments, the repeaters may actually be other idle nodes rather than special purpose devices. And second, as compared to the case when repeaters are deployed on the ceiling, the interference from repeaters towards the receivers will be significant in this case. This gives us a good lower bound on the benefits of using repeaters.

In Figure 6 we plot the average per link data rate achieved as a function of the number of links with and without repeaters. We see that when using repeaters, the average per link data rate continues to be above $1 \mathrm{Gbps}$ whereas the data rate is much lower when we do not allow repeaters. Also, the average data rate per link falls with increasing number of links because there is greater interference, even when using repeaters. Figure 7 plots the average number of repeaters used as a function of $n$ (averaged over 1,000 runs). It is interesting to see that even with $n=8$ pairs, we use an average of only 2 repeaters. But the benefits of adding these two (on average) repeaters is enormous - the average data rate jumps from less than $900 \mathrm{Mbps}$ to over $1 \mathrm{Gbps} / \mathrm{user}$.

In order to study the application of repeaters in more detail, let us consider the case when there are $n=6$ pairs. The plot for the data rate in Figure 8 shows the expected improvement in data rate per link when using repeaters. The $\mathrm{x}$-axis reports on the number of degraded links (when all pairs use the direct

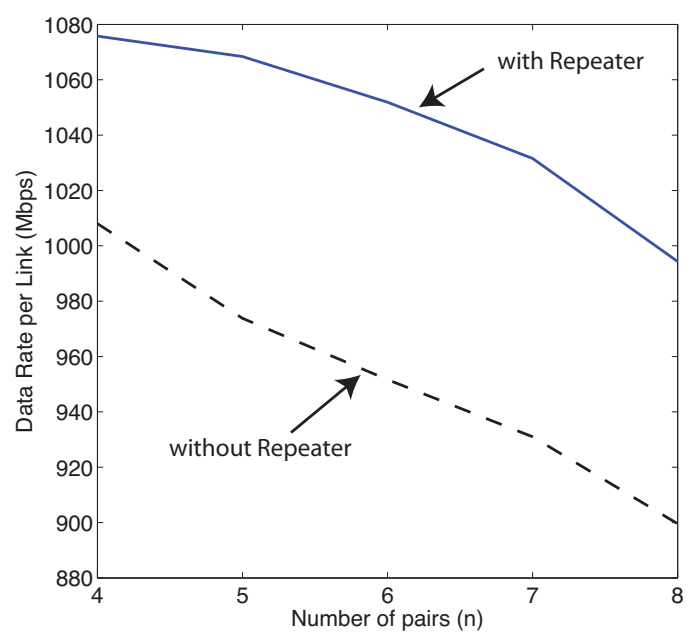

Fig. 6. Overall improvement in data rates with repeaters.

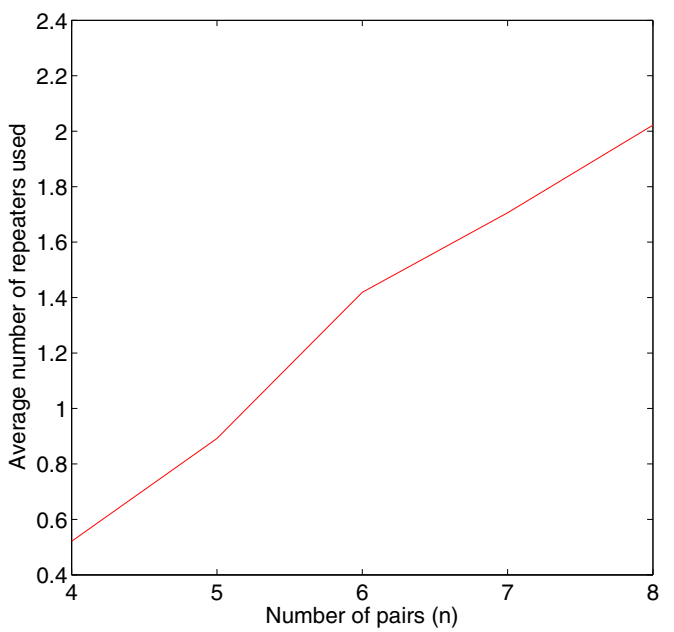

Fig. 7. Average number of repeaters used.

LoS link). When no link is degraded the data rate seen by each pair is over 1Gbps. When one pair's link is subject to interference, the average data rate without repeaters falls to $930 \mathrm{Mbps}$. But when repaired using a repeater, the data rate climbs to over $1 \mathrm{Gbps}$. When 4 or 5 of the six pairs see link degradation due to interference, the average data rate is at about $500 \mathrm{Mbps}$ only but jumps up to $1 \mathrm{Gbps}$ with repeaters.

In order to understand how often links degrade, Figure 9 plots the pdf (probability density function) of the number of links that fall below threshold when repeaters are not used. $30 \%$ of the time we see that repeaters are not required since no pair sees degraded link quality. However, about 35\% of the time one pair does see poor quality of its direct link. Interestingly, there are cases when 5 out of 6 links fall below threshold. This clearly underscores the impact of interference and the need for repeaters. Figure 10 plots the number of repeaters used as a function of the number of links that 


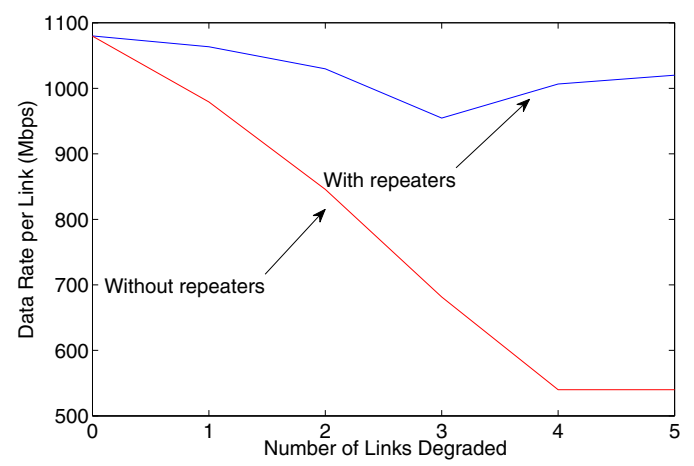

Fig. 8. Improvement in data rate for $n=6$ links.

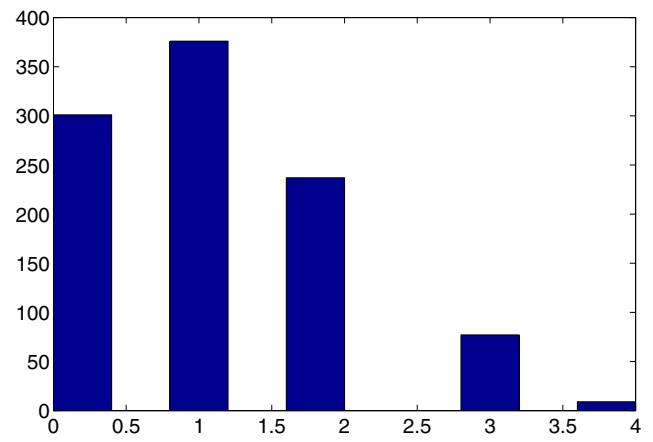

Fig. 9. Histogram of number of links broken for $n=6$ links.

degrade. The interesting observation is that the number of repeaters used scales linearly with the number of degraded links - this means that in most cases, repairing one link does little to improve another link's performance and thus each degraded link needs its own repeater. In some cases, for instance when 4 links are broken, the average number of repeaters used is 4.5. The reason for this is that re-routing a broken link via a repeater tends to break a previously good link (as we see in Figure 5 where link 5 was originally in good condition but then gets degraded due to link 4 being rerouted). Therefore, the total number of repeaters we may use could exceed the number of broken links without repeaters. In all cases, the improvement is over 50\% thus, again, showing the benefits and need to use repeaters.

\section{CONCLUSIONS}

This paper considers the problem of maintaining high data rate connectivity between pairs of communicating nodes in a $60 \mathrm{GHz}$ network. The challenge in maintaining these good connections is link breakage due to interference or mobile obstructions. We solve the problem by using repeaters that are randomly deployed about the room. If a pair of communicating nodes sees their data rate drop, they re-route their connection via a repeater. We show that a distributed greedy algorithm suffices to bring the system to a stable operating point in most cases with all pairs achieving over $1 \mathrm{Gbps}$ data rates. The next problem we are studying is integration of the link

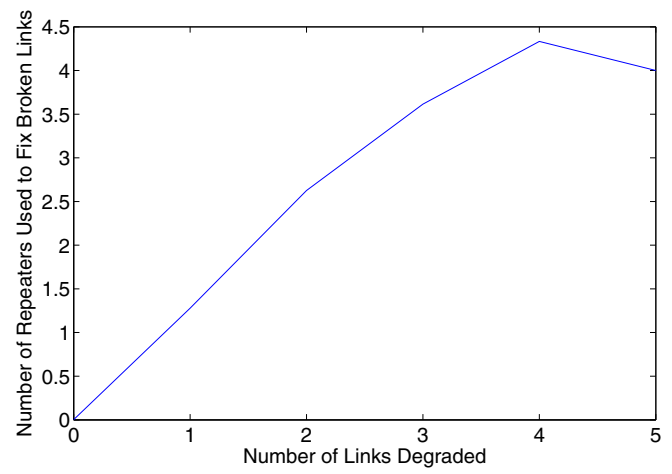

Fig. 10. Number of repeaters used for $n=6$ links.

and rate selection algorithm into MAC protocols that are under development.

\section{REFERENCES}

[1] S. E. Alexander and G. Pugliese. Cordless communications within buildings: Results and measurements at $900 \mathrm{mhz}$ and $60 \mathrm{ghz}$. British Telecom Technology Journal, 44(10):99 - 105, Oct. 1996.

[2] C. R. Anderson and T. S. Rappaport. In-building wideband partition loss measurements at 2.5 and $60 \mathrm{ghz}$. IEEE Trans. on Wireless Communications, 3(3):922 - 928, May 2004.

[3] John C. Bicket. Bit-rate selection in wireless networks. Master's thesis, MIT, 2005.

[4] J-P. Ebert et. al. Paving the way for gigabit networking. In Global Communications Newsletter, April 2005.

[5] G. Fettweis and R. Irmer. Wigwam: system concept development for 1 gbit/s air interface. Wireless OWrld, Rsearch Forum, 2005.

[6] E. Grass, M. Piz, F. Herzel, and R. Kraemer. Draft phy proposal for $60 \mathrm{ghz}$ wpan: Ieee p802.15 wg on wpans, November 2005.

[7] Frank Gross. Smart Antennas for Wireless Communications. McGraw Hill, 2005.

[8] C. P. Lim, R. J. Burkholder, J. L. Volakis, and R. J. Marhefka Propagation modeling of indoor wireless communications at $60 \mathrm{ghz}$. In IEEE Antennas and Propagation Society International Symposium, pages 2149 - 2152, 2006.

[9] M. Marcus and B. Pattan. Millimeter wave propagation: spectrum management implications. IEEE Microwave Magazine, June 2005.

[10] S. Singh, F. Ziliotto, U. Madhow, E. M. Belding, and M. J. W. Rodwell. Millimeter wave wpan: Cross-layer modeling and multi-hop architecture. In IEEE INFOCOM (Minisymposium), pages 2336 - 2340, May 2007.

[11] Bernard Sklar. Digital Communications. Prentice Hall, 2005.

[12] P. Smulders. Exploiting the $60 \mathrm{ghz}$ band for local wireless multimedia access: prospects and future directions. IEEE Communications Magazine, pages $140-147$, January 2002.

[13] S. H. Y. Wong, H. Yang, S. Lu, and V. Bharghavan. Robust rate adaptation for 802.11 wireless networks. In ACM MOBICOM, pages 146-157, 2006.

[14] H. Xu, V. kukshya, and T. Rappaport. Spatial and temporal characteristics of $60 \mathrm{ghz}$ indoor channels. IEEE Journal in Selected Areas in Communications, 20(3):620 - 630, April 2002.

[15] Candy Yiu and Suresh Singh. Empirical capacity of mmwave wlans. IEEE Journal on Selected Areas in Communication, 27(8):1479-1487, October 2009. 